\title{
An accurate performance analysis of an FFR scheme in the downlink of cellular systems under large-shadowing effect
}

\author{
Benoit Pijcke ${ }^{1,2,3}$, Marc Gazalet ${ }^{1,2,3}$, Marie Zwingelstein-Colin ${ }^{1,2,3^{*}}$ and François-Xavier Coudoux ${ }^{1,2,3}$
}

\begin{abstract}
We address the problem of analyzing the performances of interference-limited cellular networks in large-scale shadowing environments. Focusing on the fractional frequency reuse (FFR) framework, we examine how to optimally assign mobile users in a cell either to the full frequency reuse part or to the orthogonal part of the FFR band. Instead of using traditional Monte Carlo simulations, which do not provide sufficiently accurate results under important shadowing, we propose a fast and accurate numerical method. We consider a fast-fading environment and we use the ergodic capacity as the performance measure. Applying a distributed power control and scheduling strategy, we examine both cases where access points have knowledge of partial- or full-channel state information (CSI); for the latter, we also propose an approximated waterfilling strategy. The interest of our method lies in the fact that it allows for a fast and accurate analysis of the performances of FFR. In addition, it takes into account a broad range of shadowing environments.
\end{abstract}

Keywords: Wireless networks, Intercell interference, Fractional frequency reuse, Rayleigh fading, Lognormal shadowing, Ergodic capacity

\section{Introduction}

Since the invention of digital networks, efforts for providing higher capacity and higher quality of service have never stopped. With the emergence of new wireless networks that aim at providing multimedia service everytime and everywhere, the need for higher spectral efficiency has never been so high $[1,2]$. In order to achieve these goals, aggressive reuse of the whole frequency spectrum throughout the network is mandatory. As a consequence, today's systems have to make intensive use of intercell interference mitigation techniques, including power control, opportunistic scheduling, FFR, MIMO... [3-9].

As the term 'fractional' implies, FFR attempts to find a compromise between the full frequency reuse scenario, which allocates the entire available bandwidth to each cell but at the expense of a maximum level of intercell interference, and the partial frequency reuse scenario, which

\footnotetext{
${ }^{*}$ Correspondence: Marie.Zwingelstein-Colin@univ-valenciennes.fr

1 Université Lille Nord de France, F-59000 Lille, France

2 UVHC, IEMN/DOAE, F-59313 Valenciennes, France

Full list of author information is available at the end of the article
}

provides zero intercell interference by orthogonally sharing the bandwidth among adjacent cells [10]. The idea behind FFR has come from the observation that user terminals (UTs) within a cell are not equivalent from an intercell interference point of view: due to the path-loss effect, cell-edge users are far more sensitive to it than users located near the center of the cell.

A key aspect in FFR design is for the access points (APs) to appropriately decide, for each UT, whether it has to be scheduled within the full frequency reuse part of the FFR band or within the orthogonal part of the FFR band. In this article, we investigate this decision issue based on the knowledge of the users channel gains.

Generally, the performances of FFR are evaluated by Monte Carlo simulations and/or empirical measurements [8,11-13]. A recent contribution [14] has tackled the problem by following an analytical approach, however using some simplifying assumptions (very basic power control at the AP, no shadowing effect). Although the Monte Carlo simulation technique is a very common and practical tool, it does not provide sufficiently accurate results in the case where the random phenomenon

\section{Springer}

(c) 2013 Pijcke et al.; licensee Springer. This is an Open Access article distributed under the terms of the Creative Commons

Attribution License (http://creativecommons.org/licenses/by/2.0), which permits unrestricted use, distribution, and reproduction in any medium, provided the original work is properly cited. 
included in the simulation model exhibits a heavy-tailed behavior. This is due to the fact that for heavy-tailed distributions, large amplitudes are associated a very low probability, so they rarely appear in the simulation process, despite their great impact on system performance [15]. In wireless networks, the heavy-tailed behavior of the interference power originates from the cumulative effect of the shadowing phenomenon in interfering links (sum of log-normal distributions) [16,17], and this makes accurate and fast simulations very challenging in such environments.

In this article, we propose an accurate and fast numerical method to determine the performances of FFR in terms of ergodic capacity, in a multicell network suffering from path loss, shadowing and Rayleigh fading. In particular, we examine how the UT should be assigned to the FFR band, based on its channel gain. Unlike other studies that assume a fixed shadowing standard deviation (see e.g., [11,13], where $\sigma_{\mathrm{dB}}=8 \mathrm{~dB}$ ), we consider it as a variable parameter $\left(\sigma_{\mathrm{dB}} \in[0,10] \mathrm{dB}\right)$ in order to accurately analyze its influence on the performances of FFR.

The rest of the article is organized as follows. In Section 2, we present the system model. In Section 3, we derive the methodology of the numerical method that we use to provide a fast and accurate simulation of the performances of FFR. Numerical results are analyzed in Section 4.

\section{Multicell downlink transmission model}

We consider the downlink of a synchronous discrete-time 19-cell cellular network having the 2D hexagonal layout depicted in Figure 1. We assume a unit-gain omnidirectional SISO (single input, single output) antenna pattern, both for the fixed APs and the mobile UTs. The UTs are supposed to be uniformly and independently distributed over the service area. Users belonging to the same cell are considered orthogonal, which gives rise to a wireless channel primarily limited by intercell interference, especially at the cell edges (as we do not consider sectorization in this article, there is no intersector interference ${ }^{\mathrm{a}}$ ). This model corresponds, e.g., to a TDMA system. In this context, the FFR scheme that we consider is a mixture of the two following patterns for allocating the available frequency spectrum across the network:

- a partial frequency reuse pattern, denoted FR3, with reuse factor 3 ( $N=6$ interferers). For this pattern, three groups of APs are active simultaneously on three orthogonal bands;

- the full frequency reuse pattern, denoted FR1, where all APs in the network transmit at the same time using the whole frequency band ( $N=18$ intercell interferers).

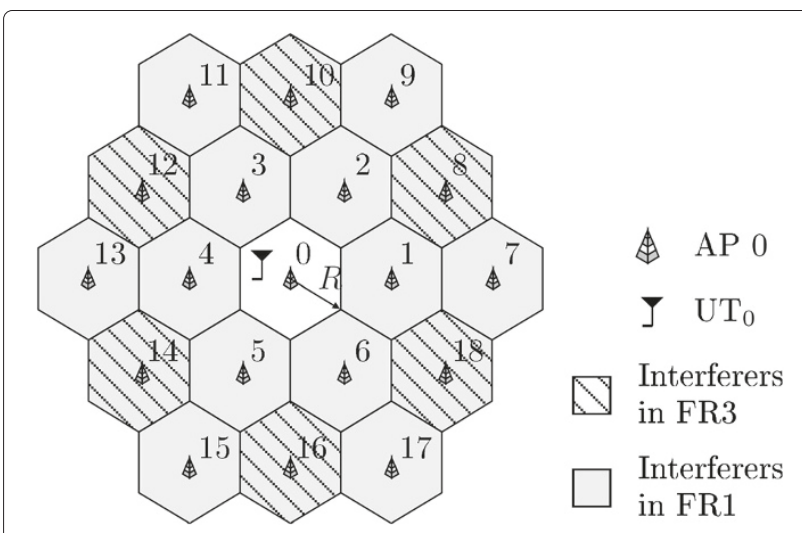

Figure 1 Cellular network model. We analyze the network performances of the typical user UT, belonging to the central cell. Two frequency reuse patterns are considered: the FR1 pattern in which all cells are active simultaneously; and the FR3 pattern, in which three groups of access points are active simultaneously on three orthogonal bands (e.g., cells $8,10,12,14,16$, and 18 are simultaneously using the same frequency band).

In this intercell-interference framework, we analyze the network performances by focusing on a typical UT. In this regard, we consider UT in cell 0 (denoted $\mathrm{UT}_{0}$, see Figure 1), because it is surrounded by 18 potential interfering cells. For $\mathrm{UT}_{0}$, the received signal at time slot $m$ can be modeled as

$$
y_{0}(m)=h_{0}(m) x_{0}(m)+\sum_{n=1}^{N} h_{n}(m) x_{n}(m)+w_{0}(m) .
$$

Here $x_{0}(m)$ represents the information symbol intended to $\mathrm{UT}_{0}$ and $x_{n}(m), n \neq 0$, the $n$th interfering symbol (this symbol is sent from AP $n$ to its respective user $\left.\mathrm{UT}_{n}\right)$. The coefficient $h_{n}(m)$ denotes the instantaneous channel gain from AP $n$ to $\mathrm{UT}_{0}$. Each channel is subject to additive white Gaussian noise $w_{0}(m)$, with variance $W$.

Each channel is assumed to be flat-fading, possibly experiencing small-scale multipath fading and/or largescale shadowing random processes. From now on, we use the instantaneous channel power gain (or simply channel gain $^{\mathrm{b}}$ ) defined by $H_{n}=\left|h_{n}(m)\right|^{2}$, that can be expressed as a three-factor product:

$$
H_{n}=H_{\mathrm{pl}, n} H_{\mathrm{f}, n} H_{\mathrm{s}, n}, \quad n=0,1, \ldots, N .
$$

Note that $H_{0}$ is the channel gain from AP 0 to $\mathrm{UT}_{0}$ (useful link), whereas $H_{1}, H_{2}, \ldots$, and $H_{N}$ represent interfering channel gains. In (1), the (deterministic) path loss $H_{\mathrm{pl}, n}$ diminishes with the distance $r_{n}$ between $\mathrm{UT}_{0}$ and $\mathrm{AP} n[18]$ :

$$
H_{\mathrm{pl}, n}=\alpha\left(\frac{1}{r_{n}}\right)^{\gamma},
$$

where $\alpha=(c /(4 \pi f))^{2} d_{0}^{\gamma-2}$, with $c$ being the speed of light, $f$, the operating frequency, and $d_{0}$, a reference 
distance for the antenna far-field; and $\gamma$ represents the attenuation coefficient. The Rayleigh fading gain $H_{\mathrm{f}, n}$ is modeled by an exponential distribution with rate parameter equal to 1 , i.e., $\mathbb{E}\left\{H_{\mathrm{f}, n}\right\}=1$. The shadowing gain $H_{\mathrm{s}, n}$ is modeled by a unit-mean lognormal distribution parameterized by what is called the shadowing standard deviation $\sigma_{\mathrm{dB}}$ (usually expressed in $\mathrm{dB}$ ) which captures the importance of the shadowing phenomenon (e.g., a $\sigma_{\mathrm{dB}}=0 \mathrm{~dB}$ scenario represents a wireless channel subject to fading only). Since r.v's $H_{\mathrm{f}, n}$ and $H_{\mathrm{s}, n}$ are independent of each other, and since $\mathbb{E}\left\{H_{\mathrm{f}, n}\right\}=\mathbb{E}\left\{H_{\mathrm{s}, n}\right\}=1$, from (1) we have $\mathbb{E}\left\{H_{n}\right\}=H_{\mathrm{pl}, n}$, which reflects the fact that the Rayleigh fading and shadowing components cause the actual gain $H_{n}$ to fluctuate about its mean value $H_{\mathrm{pl}, n}$.

We now introduce the power control and scheduling strategy that we consider in this article. In what follows, we first assume a distributed environment, i.e., each AP has access to some (local) CSI provided by its respective mobile users only. According to what type of CSI data are fed back from UTs to their serving APs, we consider two different scenarios: a partial (statistical) CSI scheme (for which only path losses are available at the APs), and a full-CSI scheme (APs have knowledge of instantaneous channel gains). Since channel information is available only at the cell level ${ }^{\mathrm{c}}$, we consider a distributed power control and scheduling strategy. A channel inversion-type power control is applied within each cell, based on the mean information (i.e., the path losses $H_{\mathrm{pl}, n}$ ) available at the APs: the power emitted at each time slot by AP $n$ is $P_{n}=$ $\mathbb{E}\left\{\left|x_{n}(m)^{2}\right|\right\}=\min \left(P_{\max }, P^{*} / H_{\mathrm{pl}, n}\right)$, where $P_{\max }$ is the maximum power available at the $\mathrm{AP}$, and $P^{*}$ is the target power at the mobile user. To this power control scenario we associate the resource-fair opportunistic scheduling policy proposed (and proved to be sum-rate optimal) in [19]: each cell ranks its users by (say decreasing) order of channel gain and assigns the best users to the available time slots, regardless of channel gains in other slots.

\section{Methodology}

In this section, we aim at developing a fast and accurate method for analyzing the performances of interferencelimited cellular systems. In this context, we focus on the FFR framework for which we examine how to assign mobile users either to the FR1 or to the FR3 band, based on their path losses (because this leads to a distributed strategy). Considering a fast-fading environment, we choose the ergodic capacity as the performance measure, and we are primarily interested in determining a potential point of intersection between the ergodic capacity curve related to FR1 and the ergodic capacity curve related to FR3 (this is illustrated in Figure 2). Or, stated otherwise, we want to answer the questions: when (where) to swap from applying FR1 to applying FR3, and how does the intersection point progress as a function of

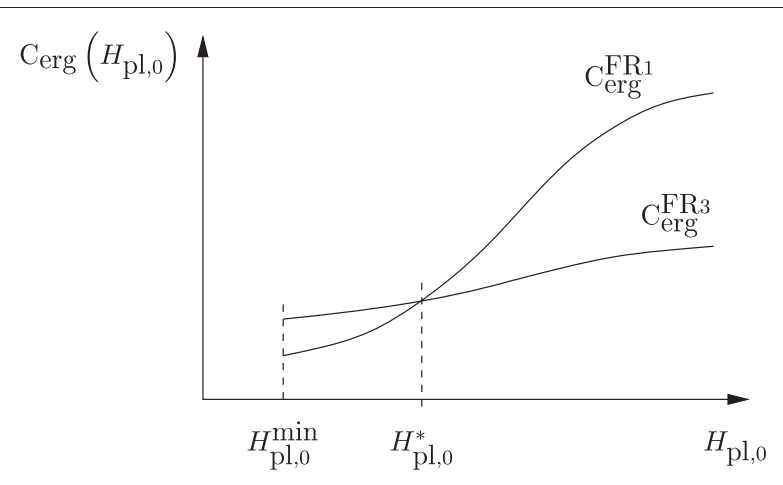

Figure 2 Plots of the ergodic capacities for both frequency reuse patterns FR1 and FR3 as functions of $\boldsymbol{H}_{\mathrm{pl}, \mathbf{0}}$. We see that for high channel gains (i.e., near the center of the cell), we obviously have $C_{\mathrm{erg}}^{\mathrm{FR} 1}>C_{\mathrm{erg}}^{\mathrm{FR} 3}$, whereas for low gains (at the cell border), we might have $C_{\mathrm{erg}}^{\mathrm{FR} 1}<C_{\mathrm{erg}}^{\mathrm{FR} 3}$. So, the ergodic capacity curves may exhibit a point of intersection $H_{\mathrm{pl}, 0}^{*}$ around which applying either FR1 or FR3 should prevail. $H_{\mathrm{pl}, 0}^{\mathrm{min}}$ is the path loss at the cell border.

the shadowing standard deviation $\sigma_{\mathrm{dB}}$ ? In addition, we want to determine the average gain when considering FR3 instead of FR1 close to the cell border, where mobile users suffer from severe interference. We also recall that we assume a distributed environment, in which APs only have access to CSI provided by their respective users.

In Section 3.1, we present the ergodic capacity as the performance measure. In Section 3.2, we consider the partial-CSI scheme. We examine the full-CSI scheme in Section 3.3, where we propose an approximated waterfilling-in-time strategy.

\subsection{The ergodic capacity as the performance measure}

We can write the ergodic capacity of the typical user $\mathrm{UT}_{0}$ as follows:

$$
C_{\mathrm{erg}}=\mathbb{E}\left\{\log _{2}\left(1+\frac{P_{0} H_{0}}{W+I}\right)\right\},
$$

where $I$ is the total interference power received by $\mathrm{UT}_{0}$, that can be written $I=\sum_{n=1}^{N} P_{n} H_{n}$. Since no closedform expression exists for the ergodic capacity (3), it must be computed numerically. The traditional approach consists in performing Monte Carlo simulations. However, because the shadowing phenomenon exhibits a heavytailed behavior for large values of the shadowing standard deviation $\sigma_{\mathrm{dB}}$, efficient simulations in such environments are very challenging. Hence the need for a method to quickly and accurately compute the ergodic capacity that takes into account the importance of the shadowing.

To propose such a method, let us closely examine (3). First of all, we investigate the impact of the power control and scheduling policy on the interference power gain $I$. Recall that the distributed scheduling policy consists, for each cell, in ranking its users by decreasing order of 
mean channel gain $H_{\mathrm{pl}, n}$, and in assigning them, in that order, an available time slot [19]. We now make an important assumption over the transmit power. Because of the scheduling policy, each user has a mean channel gain $H_{\mathrm{pl}, n}$ contained in a range which (statistically) becomes more and more restricted as the number of users per cell grows. In this article, we only consider a framework in which the number of users per cell is high. So, applying the scheduling policy described previously, it can be expected that the channels between users scheduled at the same time and their serving APs have about the same power gains: $H_{\mathrm{pl}, n} \approx H_{\mathrm{pl}, m}, \forall n \neq m$ [16]. Since the power control is based on a constant target (received) power $P^{*}$, we can reasonably assume that, at each time slot, each AP transmits at the same power: $P_{n}=P, \forall n$. In Appendix 1, we evaluate how this transmit power assumption is impacted by the total number of users.

The interference power thus simplifies to $I=$ $P \sum_{n=1}^{N} H_{n}$. We now define the interference power gainwhich is denoted $G$-as being the sum of the channel power gains between the interested user $\mathrm{UT}_{0}$ and the $N$ interferers, i.e., $G=\sum_{n=1}^{N} H_{n}=\sum_{n=1}^{N} H_{\mathrm{pl}, n} H_{\mathrm{f}, n} H_{\mathrm{s}, n}$. In this expression, $G$ is a function of $\mathrm{UT}_{0}$ 's location through the distances $r_{n}$ (see (2)). However, it has been shown in [20] that the impact of $\mathrm{UT}_{0}$ 's position on $G$ is negligible, so $G$ can be approximated by $G \approx \sum_{n=1}^{N} \mathbb{E}\left\{H_{\mathrm{pl}, n}\right\} H_{\mathrm{f}, n} H_{\mathrm{s}, n}$ (the path loss $H_{\mathrm{pl}, n}$ of the interfering link between AP $n$ and $\mathrm{UT}_{0}$ is then replaced by a mean value). Having introduced this interference power gain, we can further write $I=P G$. To characterize $G$ 's statistical law, we make use of the closed-form expression, parameterized by $\sigma_{\mathrm{dB}}$, that has been proposed in [16]. In the next place, we evaluate the expression of the power received by $\mathrm{UT}_{0}$ (see (3)), i.e., $P_{0} H_{0}=P H_{0}$. Using (1), the gain $H_{0}$ can be decomposed as $H_{0}=H_{\mathrm{pl}, 0} H_{\mathrm{f}, 0} H_{\mathrm{s}, 0}$. Since an analytical formula is available in the literature [16] for the probability law $H_{\mathrm{fs}, 0} \triangleq H_{\mathrm{f}, 0} H_{\mathrm{s}, 0}$, we can further write: $H_{0}=H_{\mathrm{pl}, 0} H_{\mathrm{fs}, 0}$.

From the above-mentioned considerations, we can rewrite the ergodic capacity (3) of the typical user $\mathrm{UT}_{0}$ as a function of its path loss $H_{\mathrm{pl}, 0}$ :

$$
C_{\mathrm{erg}}\left(H_{\mathrm{pl}, 0}\right)=\underset{H_{\mathrm{fs}, 0}, G}{\mathbb{E}}\left\{\log _{2}\left(1+\frac{P H_{\mathrm{pl}, 0} H_{\mathrm{fs}, 0}}{W+P G}\right)\right\} .
$$

Fast and accurate calculation of (4) is made possible by the use of available analytical probability laws for both r.v.s $H_{\mathrm{fs}, 0}$ and $G$; this calculation is computed numerically by estimating the sample mean in (4). This makes our method of great interest as compared with traditional time-consuming Monte Carlo simulations.

We end our discussion over the ergodic capacity by pointing out the following. We assume that mobile users are uniformly and independently distributed within each cell. If we express the ergodic capacity as a function of the r.v. $H_{\mathrm{pl}, 0}$, we can associate it the probability density function (pdf) of the typical user $\mathrm{UT}_{0}$. This pdf has the following expression [18]:

$$
p_{H_{\mathrm{pl}, 0}}\left(H_{\mathrm{pl}, 0}\right)=K\left(\frac{1}{H_{\mathrm{pl}, 0}}\right)^{\frac{\gamma+2}{\gamma}},
$$

where $K$ is a constant. Using (4) in association with (5), we are able to determine a mean network capacity by averaging the position of mobile users in the cell: this capacity corresponds to the average network capacity when there is an infinite number of users in each cell $(U \rightarrow \infty)$. Note that, for the clarity of the illustrations, we express the mean power gain $H_{\mathrm{pl}, 0}$ in $\mathrm{dB}$; the expression of its associated pdf can be easily derived from (5).

\subsection{Partial (statistical) CSI}

We first consider the case where the local path losses are the only information available at the APs: this is what we called the partial-CSI scheme (see Section 2). Under this partial-CSI scheme, we apply the path loss-based power control policy described earlier, that is, we consider that the power emitted by AP 0 towards $\mathrm{UT}_{0}$ is $P=$ $\min \left(P_{\max }, P^{*} / H_{\mathrm{pl}, 0}\right)$ which also corresponds to the power emitted by any AP towards its respective user. Using this power control policy, the ergodic capacity (4) becomes:

$$
C_{\mathrm{erg}}\left(H_{\mathrm{pl}, 0}\right)=\underset{H_{\mathrm{fs}, 0}, G}{\mathbb{E}}\left\{\log _{2}\left(1+\frac{\min \left(P_{\max }, \frac{P^{*}}{H_{\mathrm{pl}, 0}}\right) H_{\mathrm{pl}, 0} H_{\mathrm{fs}, 0}}{W+\min \left(P_{\max }, \frac{P^{*}}{H_{\mathrm{p}, 0}}\right) G}\right)\right\} .
$$

Again, we can see the great interest of our numerical method: the mathematical expectation (6) can be calculated quickly and accurately-by numerical computation of the mean in (6)-because we have at our disposal, for both frequency reuse patterns FR1 and FR3, analytical expressions for the probability laws of the r.v.s $H_{\mathrm{fs}, 0}$ and $G$, parameterized by the shadowing standard deviation $\sigma_{\mathrm{dB}}$ [16]. Plotting the ergodic capacities for FR1 and FR3, namely $C_{\mathrm{erg}}^{\mathrm{FR} 1}$ and $C_{\mathrm{erg}}^{\mathrm{FR} 3}$, as functions of $H_{\mathrm{pl}, 0}$, may then exhibit an intersection point, each side of which is applied one of the FR1 or FR3 frequence reuse patterns. This intersection point also helps us classify users in the appropriate FFR band.

Using the ergodic capacity (6) and the pdf (5), we can also determine what the average gain is of swapping from FR1 to FR3 near the cell border, where users suffer from severe interference. This gain can be calculated by comparing the ergodic capacity for the FR1 pattern with the ergodic capacity for the FR3 pattern, which we denote by $C_{\mathrm{erg}}^{\mathrm{FR} 1}\left(H_{\mathrm{pl}, 0}\right)$ and $C_{\mathrm{erg}}^{\mathrm{FR} 3}\left(H_{\mathrm{pl}, 0}\right)$, respectively. Finally, if we consider the FFR area, we can associate to this potential gain the ratio of users concerned with this policy; note that this last ratio grows exponentially as $H_{\mathrm{pl}, 0}$ diminishes. 


\subsection{Full-CSI: approximated waterfilling strategy}

We then consider the full-CSI scheme for which the APs have access to the instantaneous channel gains of their respective users. From the knowledge of the instantaneous channel conditions, we are able to propose a more efficient power allocation strategy in order to optimize the network capacity. We concentrate on what is known as the waterfilling-in-time strategy, since it is the optimal solution with respect to capacity optimization under an average transmit power constraint when both the AP and the mobile user have perfect and instantaneous knowledge of channel conditions [21].

The waterfilling strategy considered here is subject to the same path loss-based power control policy that we have used for the partial-CSI scheme, i.e., the average power received by mobile users is assumed to be constant. Under this power control and scheduling policy, the waterfilling problem performed on a per-cell basis for the full-CSI scheme can be formulated as follows:

$$
\begin{aligned}
& \max _{P_{0}}\left(\log _{2}\left(1+\frac{P_{0} H_{\mathrm{pl}, 0} H_{\mathrm{fs}, 0}}{W+\sum_{n=1}^{N} P_{n} H_{n}}\right)\right) \\
& \text { s.t. } \mathbb{E}\left\{P_{0}\right\} \leq \min \left(P_{\max }, \frac{P^{*}}{H_{\mathrm{pl}, 0}}\right),
\end{aligned}
$$$$
P_{0} \geq 0 .
$$

By using the Lagrangian method, we obtain the following solution $^{\mathrm{d}}$ :

$$
P_{0}=\left[L-\frac{W+\sum_{n=1}^{N} P_{n} H_{n}}{H_{\mathrm{pl}, 0} H_{\mathrm{fs}, 0}}\right]^{+},
$$

where the constant $L$ (the water level) satisfies the following constraint:

$$
\mathbb{E}\left\{P_{0}\right\}=\mathbb{E}\left\{\left[L-\frac{W+\sum_{n=1}^{N} P_{n} H_{n}}{H_{\mathrm{pl}, 0} H_{\mathrm{fs}, 0}}\right]^{+}\right\} .
$$

We now closely analyze the complexity of the optimization problem (7)-(9). By carefully examining (7), we highlight an important issue: the waterfilling strategy in cell 0 obviously depends upon the waterfilling procedures performed in other (interfering) cells. Indeed, the power $P_{n}$ emitted by AP $n$ results from the waterfilling strategy of cell $n$, and it therefore depends on the instantaneous channel gain $H_{\mathrm{fs}, n}$ between AP $n$ and $\mathrm{UT}_{n}$. However, since we have chosen for a distributed power control and scheduling policy, a cell only has access to local information, namely, the actual channel gains of its own users. As such, the optimization problem (7)-(9) thus reveals too complex to solve and the waterfilling strategy needs to be approximated.

For this purpose, we first assume that each r.v. $P_{n}, n=$ $1, \ldots, N$, may be assimilated to its mean value: $P_{n} \approx$ $\mathbb{E}\left\{P_{n}\right\}=\mathbb{E}\left\{P_{0}\right\}$, where the last equality results from the fact that, because of the opportunistic scheduling policy, users scheduled at the same time have the same path losses (see Section 3.1 and Appendix 1). The interference term $\sum_{n=1}^{N} P_{n} H_{n}$ then simplifies to $\mathbb{E}\left\{P_{0}\right\} \sum_{n=1}^{N} H_{n}$. Our second assumption pertains to the actual value $\sum_{n=1}^{N} H_{n}$ of the interference gain $G$ (recall that $H_{n}=H_{\mathrm{pl}, n} H_{\mathrm{fs}, n}$ is the instantaneous channel gain between AP $n$ and $\mathrm{UT}_{0}$ ). Since a distributed system is considered (which actually corresponds to any practical implementation of a wireless network), this information is not available at cell 0; so we replace $G$ by its mean value $\mathbb{E}\{G\}$. These two assumptions lead to the following (inevitably suboptimal) waterfilling solution ${ }^{\mathrm{e}}$ :

$$
\begin{aligned}
& P_{0}\left(H_{\mathrm{fs}, 0}\right)=\left[L-\frac{J}{H_{\mathrm{fs}, 0}}\right]^{+} \\
& \mathbb{E}\left\{P_{0}\left(H_{\mathrm{fs}, 0}\right)\right\}=\mathbb{E}\left\{\left[L-\frac{J}{H_{\mathrm{fs}, 0}}\right]^{+}\right\},
\end{aligned}
$$

where $J$ is defined as follows:

$$
\begin{aligned}
J & =\frac{W+\mathbb{E}\left\{P_{0}\left(H_{\mathrm{fs}, 0}\right)\right\} \mathbb{E}\{G\}}{H_{\mathrm{pl}, 0}} \\
& =\frac{W+\min \left(P_{\max }, \frac{P^{*}}{H_{\mathrm{pl}, 0}}\right) \mathbb{E}\{G\}}{H_{\mathrm{pl}, 0}} .
\end{aligned}
$$

Armed with this approximated waterfilling policy, we proceed with the computation of the ergodic capacity, which is denoted $C_{\mathrm{erg}}^{\mathrm{WF}}$. We can write from (7):

$C_{\mathrm{erg}}^{\mathrm{WF}}\left(H_{\mathrm{p}, 0}\right)=\underset{\substack{H_{\mathrm{fs}, 0}, H_{1}, H_{2}, \ldots, H_{N} \\ P_{1}, P_{2}, \ldots, P_{N}}}{\mathbb{E}}\left\{\log _{2}\left(1+\frac{P_{0}\left(H_{\mathrm{fs}, 0}\right) H_{\mathrm{pl}, 0} H_{\mathrm{fs}, 0}}{W+\sum_{n=1}^{N} P_{n} H_{n}}\right)\right\}$,

where the expectation is taken over the r.v's $H_{\mathrm{fs}, 0}, H_{n}$ and $P_{n}, n=1,2, \ldots, N$, and the instantaneous power $P_{0}\left(H_{\mathrm{fs}, 0}\right)$ is determined by (12). But we are facing exactly the same issue as before, because the power $P_{n}$ emitted by AP $n$ depends on the waterfilling policies performed in cells $m$, $m=0,1, \ldots, N, m \neq n$. Since computing the ergodic capacity (16) seems out of reach, we here too propose to assimilate $P_{n}$ to $\mathbb{E}\left\{P_{n}\right\}=\mathbb{E}\left\{P_{0}\right\}$. We show in Appendix 2 that introducing that approximation allows us to establish 
a lower bound for the ergodic capacity (16). This lower bound is denoted $C_{\mathrm{erg}}^{\mathrm{wf}}$ and it has the following expression:

$$
C_{\mathrm{erg}}^{\mathrm{wf}}\left(H_{\mathrm{pl}, 0}\right)=\underset{H_{\mathrm{f}, 0}, G}{\mathbb{E}}\left\{\log _{2}\left(1+\frac{\left[L H_{\mathrm{fs}, 0}-J\right]^{+} H_{\mathrm{pl}, 0}}{W+\min \left(P_{\max }, \frac{P^{*}}{H_{\mathrm{pl}, 0}}\right) G}\right)\right\},
$$

where $L$ and $J$ are both functions of $H_{\mathrm{pl}, 0}$. As mentioned before, our method here also allows for a fast and accurate (numerical) computation of the ergodic capacity (17) because the probability laws of $H_{\mathrm{fs}, 0}$ and $G$ can be expressed analytically [16].

We end this section by formulating some concluding remarks. From Sections 3.2 and 3.3, we can establish the following inequality:

$$
C_{\mathrm{erg}}\left(H_{\mathrm{pl}, 0}\right) \leq C_{\mathrm{erg}}^{\mathrm{wf}}\left(H_{\mathrm{pl}, 0}\right) \leq C_{\mathrm{erg}}^{\mathrm{WF}}\left(H_{\mathrm{pl}, 0}\right),
$$

where the ergodic capacities $C_{\mathrm{erg}}\left(H_{\mathrm{pl}, 0}\right)$ and $C_{\mathrm{erg}}^{\mathrm{wf}}\left(H_{\mathrm{pl}, 0}\right)$ can be computed easily, respectively, from (6) and (17) (computation of the optimal value $C_{\mathrm{erg}}^{\mathrm{WF}}\left(H_{\mathrm{pl}, 0}\right)$ is intractable and meaningless in a realistic scenario). And, as for the partial-CSI scheme, we are interested in determining a potential point of intersection of the capacity curves $C_{\mathrm{erg}}^{\mathrm{wf}, \mathrm{FR} 1}\left(H_{\mathrm{pl}, 0}\right)$ and $C_{\mathrm{erg}}^{\mathrm{wf}, \mathrm{FR} 3}\left(H_{\mathrm{pl}, 0}\right)$, for both the frequency reuse patterns FR1 and FR3, and for different values of the shadowing standard deviation $\sigma_{\mathrm{dB}}$. This intersection point also allows us to assign mobile users either to the FR1 band or to the FR3 band on the basis of their path losses.

\section{Numerical results}

In this section, we present numerical results related to the analysis of the FFR framework under different frequency reuse scenarios and for different values of the shadowing standard deviation. In Section 4.1, we first describe the simulation parameters. In Section 4.2, we compare results of the ergodic capacity as a function of the path loss under both the FR1 and FR3 patterns and we compute the potential gain of applying the FFR technique in different wireless channels. In Section 4.3, we improve the ergodic capacity results by performing the approximated waterfilling policy described in Section 3.2.

\subsection{Simulation parameters}

We use the following simulation parameters. We consider a system functioning at $1 \mathrm{GHz}$. We fix the cell radius to $R=700 \mathrm{~m}$, the far-field distance to $d_{0}=10 \mathrm{~m}$, and the attenuation coefficient to $\gamma=3.2$, which corresponds to a typical urban environment, as described by the COST231 reference model [22]. We consider a wireless channel that is subject to a large-scale shadowing phenomenon that may vary in a $[0,10] \mathrm{dB}$ range. The maximum power available at the access point is $P_{\max }=5 \cdot 10^{-3} \mathrm{~W}$ and the target received power is $P^{*}=10^{-12} \mathrm{~W}$. The additive white Gaussian noise has variance $W=10^{-15} \mathrm{~W}$. As stated previously (see Section 3.1), the number of users per cell is considered to be infinite.

\subsection{Partial-CSI scheme}

We now present results in the case of partial CSI available at the APs. First of all, we examine how the ergodic capacity of the typical user evolves as a function of its path loss, which is illustrated in Figure 3 for both frequency reuse patterns FR1 and FR3, and for several values of the shadowing standard deviation $\left(\sigma_{\mathrm{dB}}=\{0,5,10\} \mathrm{dB}\right)$; all capacity curves are associated the same pdf (plotted on the bottom figure). A careful examination of these curves allows us to highlight some interesting points. First, we see that both ergodic capacities $C_{\mathrm{erg}}^{\mathrm{FR} 1}\left(H_{\mathrm{pl}, 0}\right)$ and $C_{\mathrm{erg}}^{\mathrm{FR} 3}\left(H_{\mathrm{pl}, 0}\right)$ are constant for high path losses (i.e., close to the AP). This can be easily explained: users located near the AP suffers from less interference, which is reinforced by the power control and scheduling policy applied by each cell (users scheduled at the same time have similar gains). Since in that fully power controlled area the interference power can be neglected $(I \ll W)$, the ergodic capacity (6) becomes

$$
C_{\mathrm{erg}}\left(H_{\mathrm{pl}, 0}\right) \approx \underset{H_{\mathrm{fs}, 0}}{\mathbb{E}}\left\{\log _{2}\left(1+\frac{P^{*} H_{\mathrm{fs}, 0}}{W}\right)\right\},
$$

and it depends only on $\sigma_{\mathrm{dB}}$ (through the r.v. $\left.H_{\mathrm{fs}, 0}\right)$. Moreover, in this noise-limited scenario for which $I \rightarrow 0$ (which in the context of this article occurs when $H_{\mathrm{pl}, 0} \rightarrow \infty$ ), we can write

$$
\lim _{H_{\mathrm{pl}, 0} \rightarrow \infty} \frac{C_{\mathrm{erg}}^{\mathrm{FR} 3}\left(H_{\mathrm{pl}, 0}\right)}{C_{\mathrm{erg}}^{\mathrm{FR} 1}\left(H_{\mathrm{pl}, 0}\right)}=\frac{1}{3},
$$

because in the FR1 scenario, the whole frequency band is used while this resource is divided in three orthogonal bands in the FR3 scenario. Next, we see that all capacity curves slightly take down on the left side of a fixed gain denoted $H_{\mathrm{pl}, 0}^{\mathrm{d}}$. This results from the maximum power constraint applied at the APs: $H_{\mathrm{pl}, 0}^{\mathrm{d}}$ is the demarcation point between the fully power controlled area (right side) and the area where APs emit at their maximum (saturated) power (left side); it follows immediately that $H_{\mathrm{pl}, 0}^{\mathrm{d}}=$ $P^{*} / P_{\max }$.

In the next place, we make observations related to the FFR technique and we evaluate its performances with respect to network capacity. We observe in Figure 3 that the ergodic capacities $C_{\mathrm{erg}}^{\mathrm{FR} 1}\left(H_{\mathrm{pl}, 0}\right)$ and $C_{\mathrm{erg}}^{\mathrm{FR} 3}\left(H_{\mathrm{pl}, 0}\right)$ intersect for values of the shadowing standard deviation up to $6 \mathrm{~dB}$. This makes the FFR technique attractive in these wireless channels and our method allows for optimally assigning users to either the FR1 band or the FR3 band 


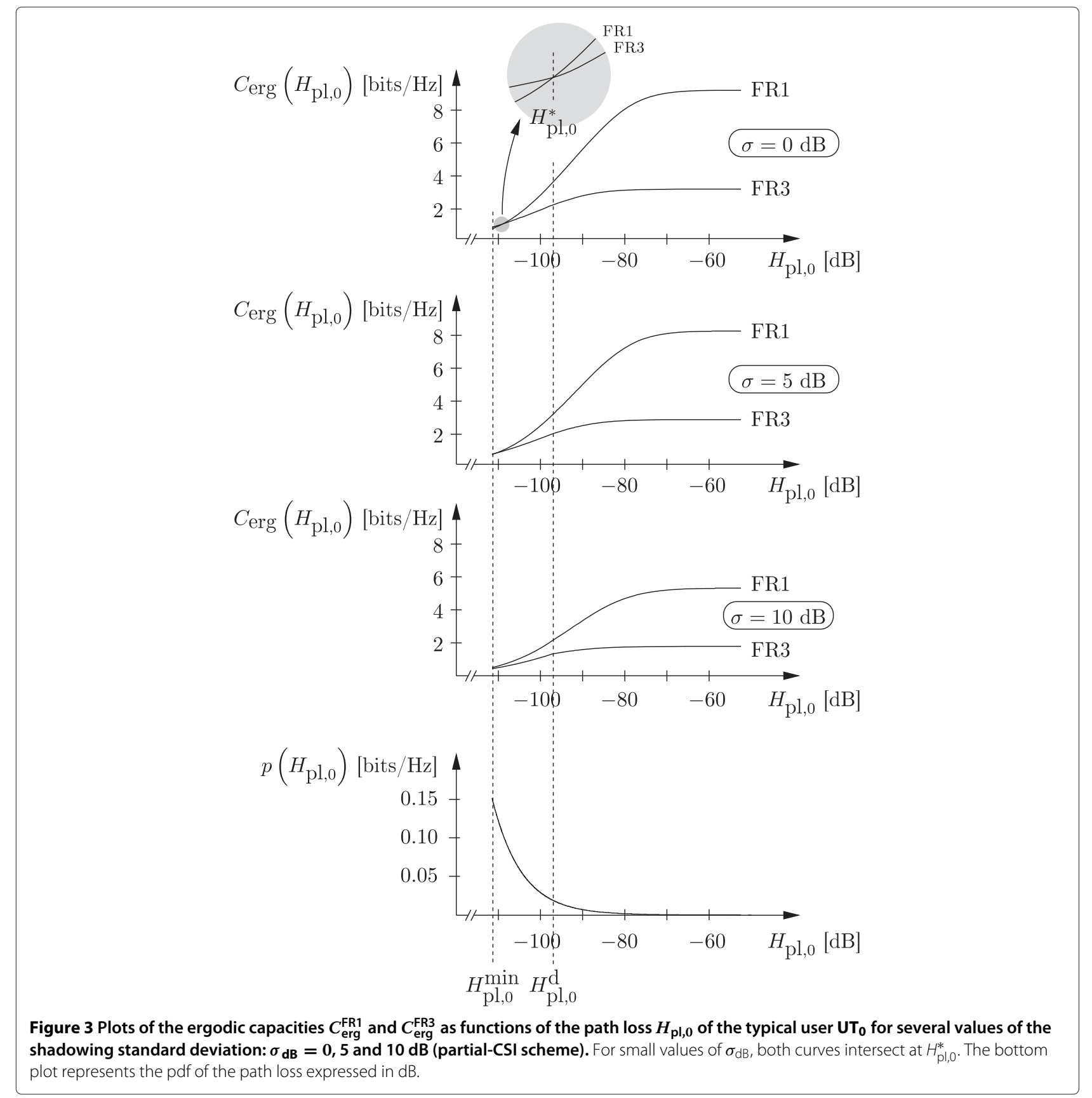

from the sole knowledge of their path losses. The evolution of the intersection point $H_{\mathrm{pl}, 0}^{*}$ as a function of $\sigma_{\mathrm{dB}}$ is plotted in Figure 4. Swapping from FR1 to FR3 when path losses are lower than $H_{\mathrm{pl}, 0}^{*}$ (i.e., for users undergoing more interference) then allows to increase the ergodic capacity. The proportion of mobile users assigned to the FR3 orthogonal band as a function of $\sigma_{\mathrm{dB}}$ can be determined by computing

$$
\int_{H_{\mathrm{pl}, 0}^{\min }}^{H_{\mathrm{pl}, 0}^{*}} p\left(H_{\mathrm{pl}, 0}\right) \mathrm{d} H_{\mathrm{pl}, 0} .
$$

This proportion is illustrated in Figure 5 and we see that it can reach up to $30 \%$ for light shadowing environments $\left(\sigma_{\mathrm{dB}} \in[0,2]-\mathrm{dB}\right.$ range). From the ergodic capacity curves and the associated probability density function, we can compute the average gain of using FR3 vs. using FR1 as follows:

$$
\frac{\int_{H_{\mathrm{pl}, 0}^{\min }}^{H_{\mathrm{m}}^{*}} C_{\mathrm{erg}}^{\mathrm{FR} 3}\left(H_{\mathrm{pl}, 0}\right) p\left(H_{\mathrm{pl}, 0}\right) \mathrm{d} H_{\mathrm{pl}, 0}}{\int_{H_{\mathrm{pl}, 0}^{\min }}^{H_{\mathrm{pl}}^{*}} C_{\mathrm{erg}}^{\mathrm{FR} 1}\left(H_{\mathrm{pl}, 0}\right) p\left(H_{\mathrm{pl}, 0}\right) \mathrm{d} H_{\mathrm{pl}, 0}}>1 .
$$




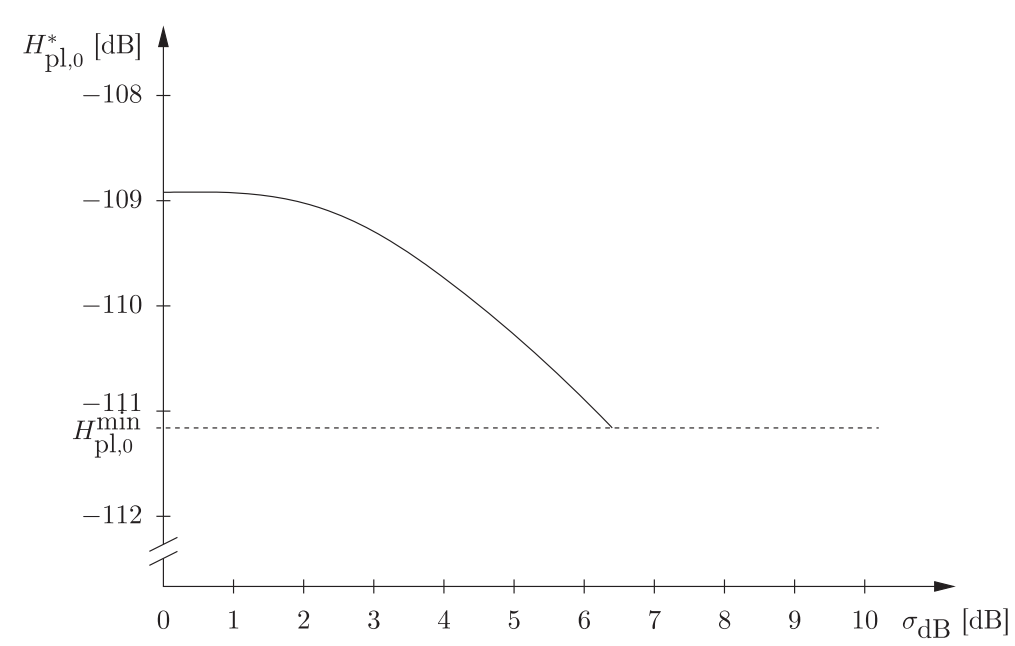

Figure 4 Plot of the intersection point $H_{\mathrm{pl}, 0}^{*}$ as a function of the shadowing standard deviation $\sigma_{\mathrm{dB}}$ for the partial-CSI scheme (values are computed from Table 1).

We summarize all the above observations for the partialCSI scheme in Table 1. Columns 2-6 show the results for the orthogonal FFR band $\left(H_{\mathrm{pl}, 0} \in\left[H_{\mathrm{pl}, 0}^{\min }, H_{\mathrm{pl}, 0}^{*}\right]\right)$. The gain in col. 5 is computed from (22) and columns 3 and 4. Results from col. 6 are calculated by (21) and have already been illustrated in Figure 5. Columns 7-9 pertain to the 10-percent proportion of mobile users located nearest to the cell border. We see that using FFR leads to a non negligible gain (at least 10\%, see col. 9) in light shadowing environments $\left(\sigma_{\mathrm{dB}}\right.$ up to $4 \mathrm{~dB}$ ). The same observation holds for the 1-percent proportion of users (columns 10-12). Our analysis confirms results obtained in other studies (for a wireless channel subject to Rayleigh fading only, see e.g., $[14,23])$.

We come to the conclusion that, for the partial-CSI scheme, the FFR technique may increase the average ergodic capacity for users located near the cell border, in light or moderate shadowing environments. But, more

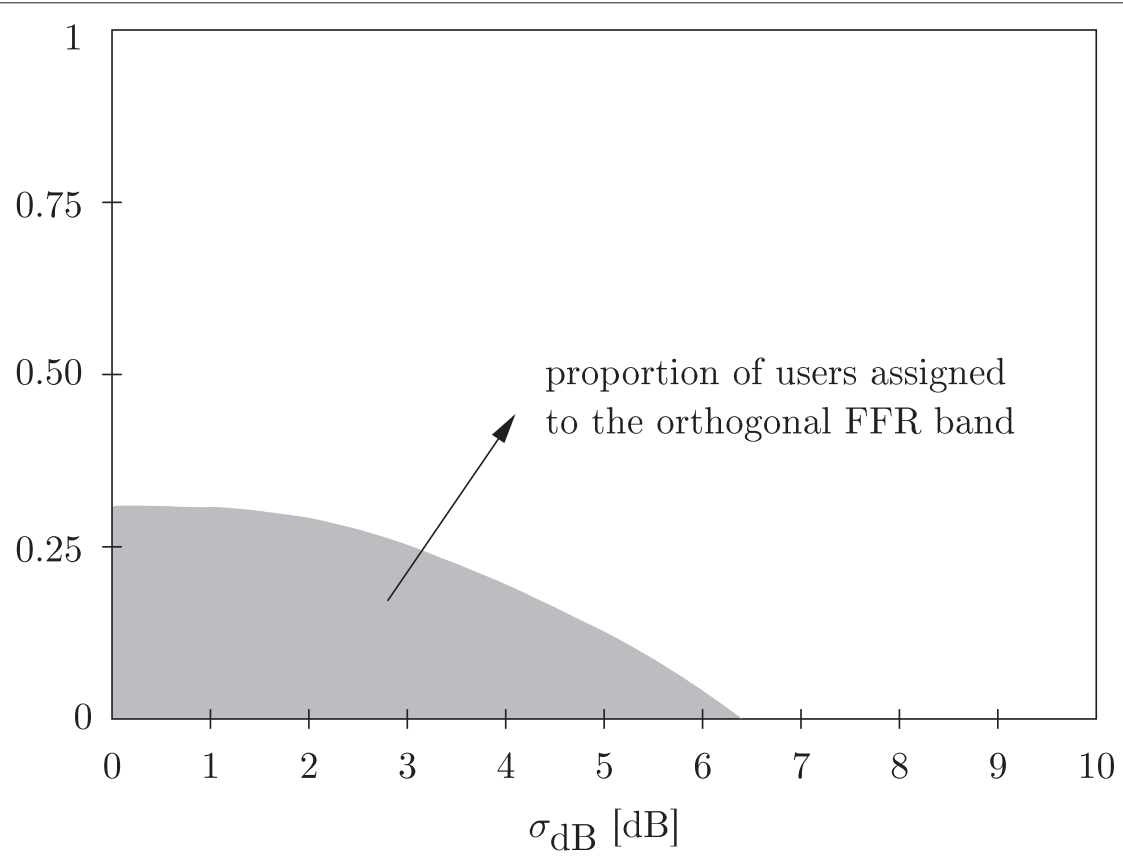

Figure 5 Plot of the proportion of mobile users assigned to the FR3 orthogonal band, as a function of the shadowing standard deviation $\sigma_{\mathbf{d B}}$ for the partial-CSI scheme (values are computed from Table 1). We see that, for light shadowing environments $\left(\sigma_{\mathrm{dB}} \in[0,2]-\mathrm{dB}\right.$ range), this proportion can reach up to $30 \%$. 
Table 1 Mean ergodic capacities $\mathbb{E}\left\{C_{\mathrm{erg}}^{\mathrm{FR} 1}\right\}$ and $\mathbb{E}\left\{C_{\mathrm{erg}}^{\mathrm{FR} 3}\right\}$ for the partial-CSI scheme and for different values of the shadowing standard deviation $\sigma_{\mathrm{dB}}$

\begin{tabular}{|c|c|c|c|c|c|c|c|c|c|c|c|}
\hline \multirow[b]{2}{*}{$\sigma_{\mathrm{dB}}$} & \multicolumn{5}{|c|}{ FFR area } & \multicolumn{3}{|c|}{$10 \%$ users } & \multicolumn{3}{|c|}{$1 \%$ users } \\
\hline & $\begin{array}{l}H_{\mathrm{pl}, 0}^{*} \\
{[\mathrm{~dB}]}\end{array}$ & $\begin{array}{r}\mathbb{E}\left\{C_{\mathrm{erg}}^{\mathrm{FR} 1}\right\} \\
{[\mathrm{bits} / \mathrm{Hz}]}\end{array}$ & $\begin{array}{r}\mathbb{E}\left\{C_{\mathrm{erg}}^{\mathrm{FR} 3}\right\} \\
{[\mathrm{bits} / \mathrm{Hz}]}\end{array}$ & $\begin{array}{c}\text { gain } \\
{[\%]}\end{array}$ & $\begin{array}{c}\text { users } \\
\text { [\%] }\end{array}$ & $\begin{array}{r}\mathbb{E}\left\{C_{\mathrm{erg}}^{\mathrm{FR} 1}\right\} \\
\text { [bits/Hz] }\end{array}$ & $\begin{array}{r}\mathbb{E}\left\{C_{\text {erg }}^{\mathrm{FR} 3}\right\} \\
{[\text { bits/Hz] }}\end{array}$ & $\begin{array}{c}\text { gain } \\
{[\%]}\end{array}$ & $\begin{array}{r}\mathbb{E}\left\{C_{\mathrm{erg}}^{\mathrm{FR} 1}\right\} \\
{[\text { bits/Hz] }}\end{array}$ & $\begin{array}{r}\mathbb{E}\left\{C_{\mathrm{erg}}^{\mathrm{FR} 3}\right\} \\
\text { [bits/Hz] }\end{array}$ & $\begin{array}{l}\text { gain } \\
{[\%]}\end{array}$ \\
\hline 0 & -108.92 & 0.737 & 0.794 & 7.730 & 30.8 & 0.639 & 0.726 & 13.61 & 0.605 & 0.701 & 15.9 \\
\hline 1 & -108.92 & 0.736 & 0.793 & 7.706 & 31.0 & 0.638 & 0.725 & 13.58 & 0.604 & 0.700 & 15.8 \\
\hline 2 & -108.99 & 0.725 & 0.777 & 7.224 & 30.2 & 0.634 & 0.714 & 12.63 & 0.600 & 0.689 & 14.8 \\
\hline 3 & -109.26 & 0.702 & 0.744 & 6.070 & 27.4 & 0.627 & 0.692 & 10.33 & 0.595 & 0.668 & 12.3 \\
\hline 4 & -109.72 & 0.670 & 0.700 & 4.440 & 22.4 & 0.621 & 0.666 & 7.11 & 0.591 & 0.643 & 8.9 \\
\hline 5 & -110.26 & 0.637 & 0.655 & 2.841 & 16.2 & 0.615 & 0.640 & 4.00 & 0.585 & 0.618 & 5.6 \\
\hline 6 & -110.88 & 0.600 & 0.607 & 1.273 & 8.4 & 0.605 & 0.611 & 1.01 & 0.577 & 0.591 & 2.4 \\
\hline 7 & & & N.A. & & & & N.A. & & & N.A. & \\
\hline 8 & & & N.A. & & & & N.A. & & & N.A. & \\
\hline 9 & & & N.A. & & & & N.A. & & & N.A. & \\
\hline 10 & & & N.A. & & & & N.A. & & & N.A. & \\
\hline
\end{tabular}

N.A. stands for not applicable.

importantly, besides this capacity increase, what really makes FFR of great interest is the fact that it may concern a non negligible proportion of mobile users, especially for small values of the shadowing standard deviation.

\subsection{Full-CSI scheme}

Finally, we consider the full-CSI scheme associated with the approximated waterfilling policy proposed in Section 3.3. The ergodic capacities $C_{\mathrm{erg}}^{\mathrm{wf}, \mathrm{FR} 1}\left(H_{\mathrm{pl}, 0}\right)$ and $C_{\mathrm{erg}}^{\mathrm{wf}, \mathrm{FR} 3}\left(H_{\mathrm{pl}, 0}\right)$, obtained from $(17)$, as well as the associated pdf $p\left(H_{\mathrm{pl}, 0}\right)$, are plotted in Figure 6 , for both frequency reuse patterns FR1 and FR3, and for $\sigma_{\mathrm{dB}}=0,5$ and $10 \mathrm{~dB}$.

We first observe that, as compared with the partial-CSI scheme (see Figure 3), the waterfilling strategy obviously improves the capacity results. We next see that the FR1 pattern performs better than the FR3 pattern, for the whole $[0,10]-\mathrm{dB}$ range of values of the shadowing standard deviation $\sigma_{\mathrm{dB}}$. Let us explain why the presence of an intersection point in the partial-CSI scheme offers no clue as to the presence of such a point in the full-CSI scheme (for the same shadowing standard deviation $\sigma_{\mathrm{dB}}$ ). Considering a partial-CSI scenario where ergodic capacity curves intersect, we can write, for $H_{\mathrm{pl}, 0}<H_{\mathrm{pl}}^{*}$,

$$
C_{\mathrm{erg}}^{\mathrm{FR} 1}\left(H_{\mathrm{pl}, 0}\right)<C_{\mathrm{erg}}^{\mathrm{FR} 3}\left(H_{\mathrm{pl}, 0}\right) .
$$

Since the waterfilling gain increases when the signal-to(interference-plus)-noise ratio decreases, (23) yields

$$
\frac{C_{\mathrm{erg}}^{\mathrm{wf}, \mathrm{FR} 1}\left(H_{\mathrm{pl}, 0}\right)}{C_{\mathrm{erg}}^{\mathrm{FR} 1}\left(H_{\mathrm{pl}, 0}\right)}>\frac{C_{\mathrm{erg}}^{\mathrm{wf}, \mathrm{FR} 3}\left(H_{\mathrm{pl}, 0}\right)}{C_{\mathrm{erg}}^{\mathrm{FR} 3}\left(H_{\mathrm{pl}, 0}\right)},
$$

where $H_{\mathrm{pl}, 0}<H_{\mathrm{pl}, 0}^{*}$. Inequality (24) is clearly illustrated in Figure 7 for $H_{\mathrm{pl}, 0}=H_{\mathrm{pl}, 0}^{\mathrm{min}}$ and $\sigma_{\mathrm{dB}}=0 \mathrm{~dB}$. But from (23) and (24), we have no indication about the validity of either $C_{\mathrm{erg}}^{\mathrm{wf}, \mathrm{FR} 1}\left(H_{\mathrm{pl}, 0}\right) \geq C_{\mathrm{erg}}^{\mathrm{wf}, \mathrm{FR} 3}\left(H_{\mathrm{pl}, 0}\right)$ or $C_{\mathrm{erg}}^{\mathrm{wf}, \mathrm{FR} 1}\left(H_{\mathrm{pl}, 0}\right) \leq$ $C_{\mathrm{erg}}^{\mathrm{wf}, \mathrm{FR} 3}\left(H_{\mathrm{pl}, 0}\right)$.

From these observations, we can conclude that applying FFR is of no interest with respect to network capacity when APs perfectly know their local channel conditions (full-CSI scheme), though other simulation scenarios may lead to different conclusions.

\section{Conclusion and future study}

In this article, we investigated the problem of evaluating the performances of FFR in large-scale shadowing environments. Instead of using Monte Carlo simulations or empirical measurements, we proposed a numerical method to easily compute the ergodic capacity in a fastfading environment. Using a distributed power control and scheduling policy, we examined both cases where APs have access to partial- or full-CSI. We showed that our method allows for a fast and accurate analysis of the performances of FFR. In addition, it takes into account a broad range of shadowing environments.

The results presented in this article only hold for the simulation conditions specified; they may differ for other simulation scenarios. So a future article will pertain to applying the proposed method to other types of network (e.g., ad hoc networks, ...) and using different simulation conditions (user distribution in the cell, transmit or target power, attenuation coefficient, ...). Another perspective is to consider sectorization, which induces intersector interference and leads to totally different models for the 


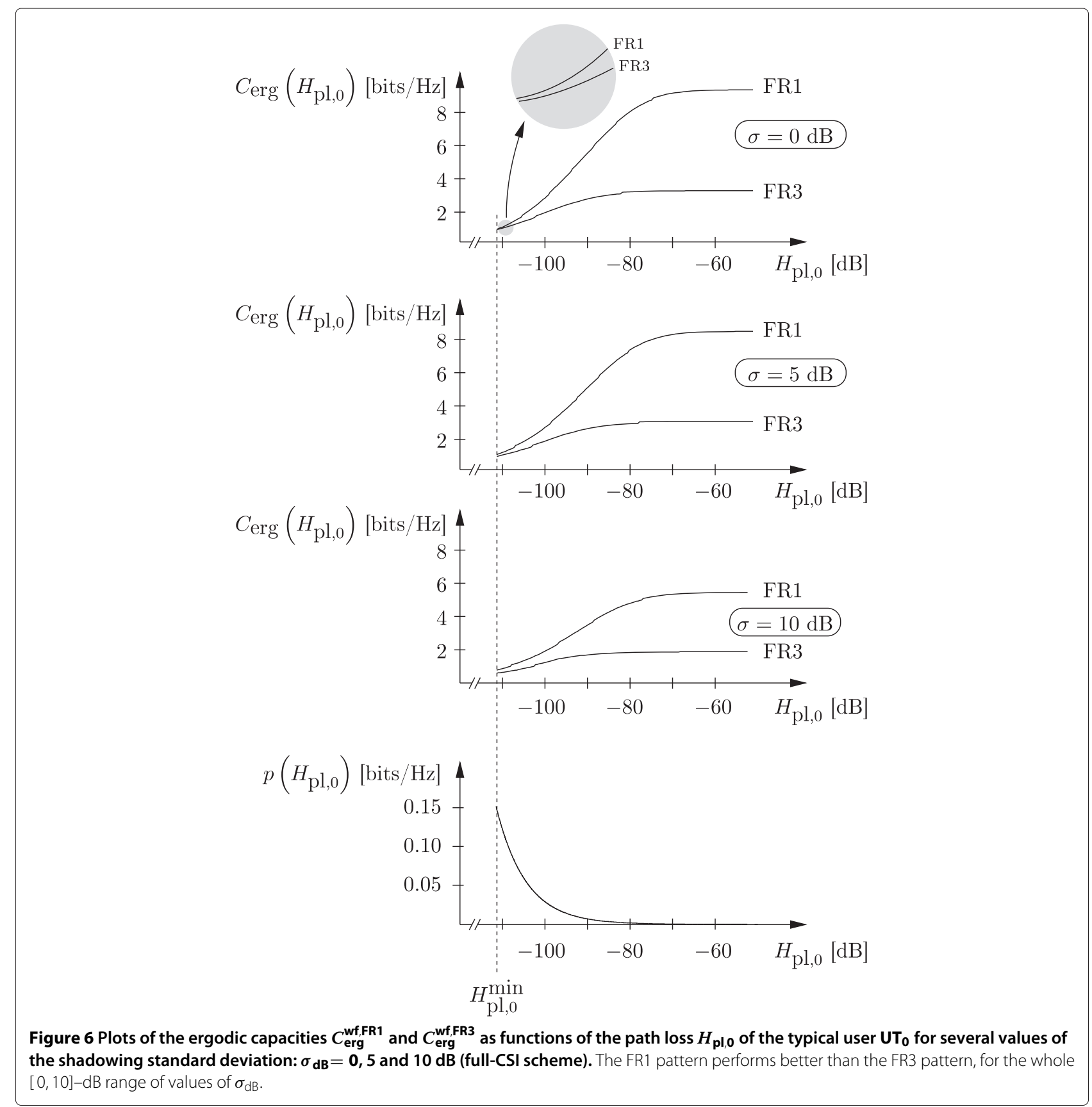

power gains as well as for the interference. Yet another perspective concerns the analysis of the network performances in a slow fading environment using other performance measures, like the outage capacity or the outage probability.

\section{Appendix 1}

Validity of the assumption over the transmit power

In this Appendix, we analyze our assumption over the transmit power. First we investigate how the total number of users impacts the transmit power within one cell and we evaluate our assumption in the case where all cells have the same number of users. Then we examine the case where the total number of users varies among cells.

\section{Same number of users per cell}

Let $U$ be the number of users in a cell, and let us assume that all users are uniformly and independently distributed over the cell area (for the sake of simplicity, we consider a circular area of radius $R$ ). Since we consider a channel inversion power control strategy, we have $P \propto 1 / H_{\mathrm{pl}}$, and 


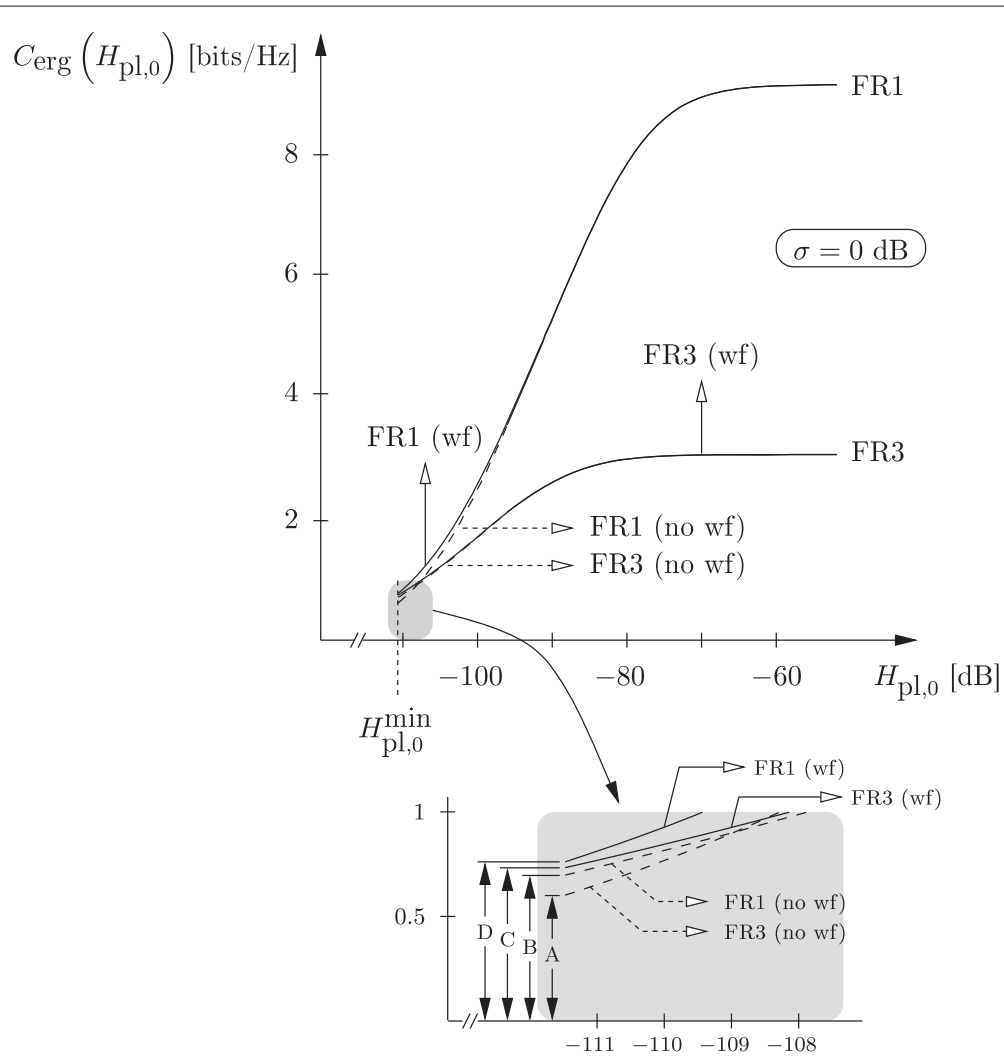

Figure 7 We illustrate the waterfilling gain for the ergodic capacity at the minimum path loss $H_{\mathrm{p}, 0}^{\min }$ for both frequency reuse schemes FR1 and FR3 (full-CSI scheme). We see from the bottom figure that $C / A>D / B$, which results from (24).

the distribution function of the transmit power $P$ can be easily written as follows:

$$
F(P)=\left(\frac{P}{P_{\max }}\right)^{2 / \gamma}
$$

where $P_{\max } \propto R^{\gamma}$ is the power transmitted to a user located at the cell border. Using order statistics (recall that all users are scheduled in decreasing order of their channel gains) [25], the pdf of user $u$ can be written

$$
f\left(P_{u}\right)=\frac{F^{u-1}\left(P_{u}\right)\left(1-F\left(P_{u}\right)\right)^{U-u} F^{\prime}\left(P_{u}\right)}{\beta(u, U-u+1)}, \quad 0 \leq P_{u} \leq 1,
$$

where $P_{u}$ is the power transmitted to user of rank $u$ (for a total of $U$ users in the cell), $F$ is defined by (25) and $\beta(x, y)=\int_{0}^{1} t^{x-1}(1-t)^{y-1} d t$ is the beta function. Some of these pdf's are illustrated in Figure 8 for different values of $U$. To evaluate our assumption over the transmit power, let us compute the standard deviation $\sigma_{P_{u}}$; this quantity can be obtained from (25) and (26):

$$
\sigma_{P_{u}}=\sqrt{\frac{\beta(u+\gamma, U-u+1)-\beta^{2}\left(u+\frac{\gamma}{2}, U-u+1\right)}{\beta(u, U-u+1)}} .
$$

Table 2 shows maximum values of the standard deviation (as a proportion of the whole $[0,1]$-dynamics) for different numbers of users in the cell. We see that large values of $\sigma_{P_{u}}$ occur for small values of $U$, and that $\sigma_{P_{u}}$ becomes smaller as $U$ increases. In a framework where all APs have the same (large) number of users, it can then be reasonably stated that users scheduled at the same time are allocated the same power, i.e., $P_{n}=P, \forall n$, at each time slot.

\section{Variability in the number of users per cell}

We now examine the case where the total number of users varies from cell to cell. To this end, we focus on the particular case of a two-cell network in which cell 1 and cell 2 contain $U_{1}=60$ users and $U_{2}=120$ users, respectively, and we consider a frame consisting of 120 time slots. In both cells, users are ranked according to their decreasing channel gains, and they are allocated time slots in that order; each user in cell 1 is then allocated two consecutive time slots. This leads to the following scheduling algorithm:

cell 1: $v_{1,1} \quad v_{1,1} \quad v_{1,2} \quad v_{1,2} \quad \ldots \quad v_{1,60} \quad v_{1,60}$

cell 2: $v_{2,1} \quad v_{2,2} \quad v_{2,3} \quad v_{2,4} \quad \ldots \quad v_{2,119} v_{2,120}$ 

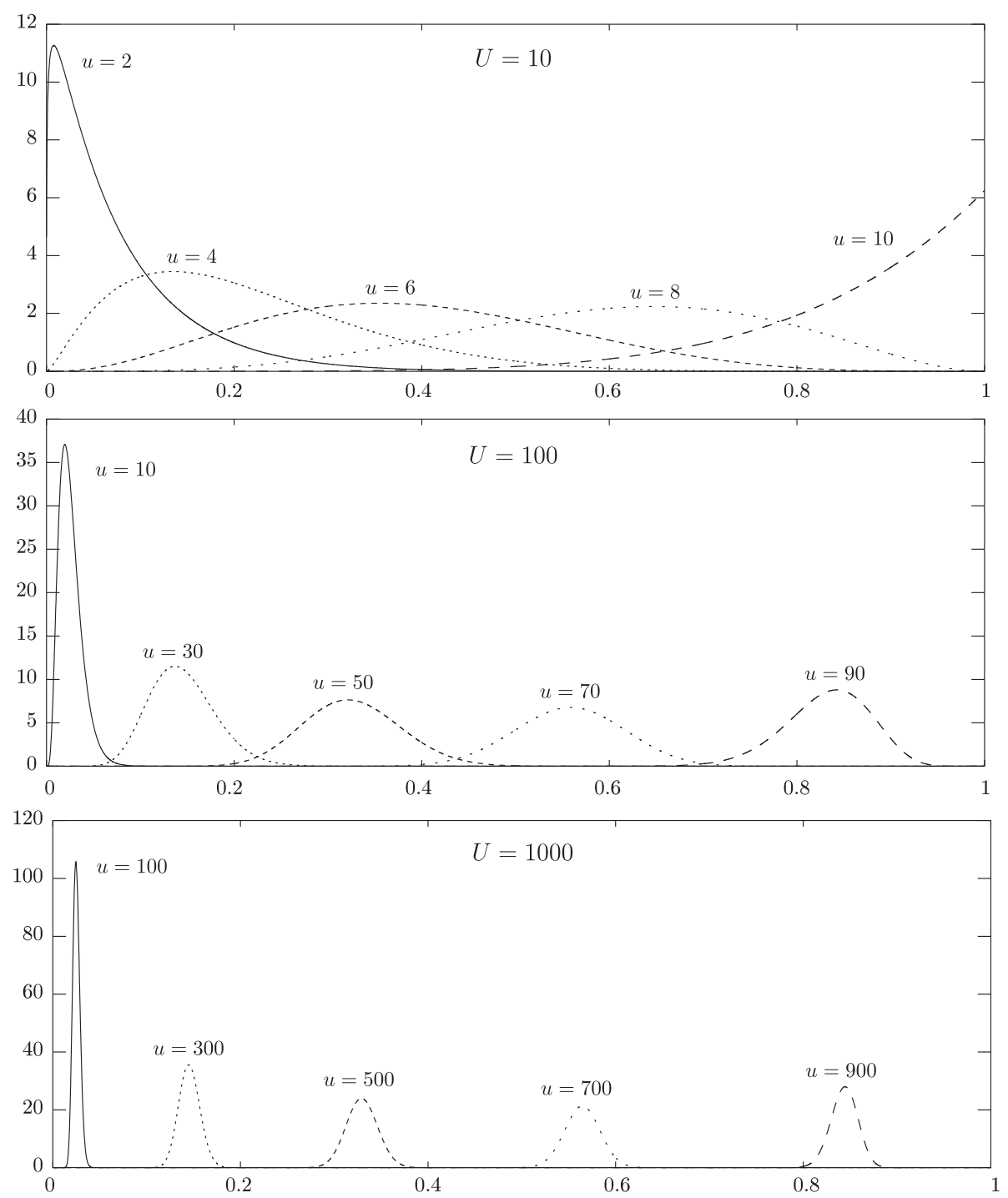

Figure 8 Some pdfs of transmit power of mobile users scheduled in decreasing order of their mean channel gain for $U=10, U=100$, and $U=\mathbf{1 0 0 0}$ users in each cell. It can be seen that standard deviations greatly diminish with $U$.

where $v_{n, u}$ represents user $u$ of cell $n$. To evaluate our assumption, we compute the ratio

$$
\Delta=\left|P_{1, t}-P_{2, t}\right| / P_{1, t},
$$

where $P_{n, t}$ is the (mean) power $\mathbb{E}\left\{P_{u}\right\}$-whose statistics is given by (26) - transmitted by AP $n$ towards user scheduled at time slot $t$ (in our case, $t=1,2, \ldots, 120$ ). Results are given in Table 3 . We see that, for more than $93 \%$ of

Table 2 Maximum values of the standard deviation $\sigma_{P_{u}}$, as a proportion of the $[0,1]$-dynamics of $P_{u}$, for a total of $U=10, U=100$, and $U=1000$ users in a cell

\begin{tabular}{cccc}
\hline & $\boldsymbol{U}=\mathbf{1 0}$ & $\boldsymbol{U}=\mathbf{1 0 0}$ & $\boldsymbol{U}=\mathbf{1 0 0 0}$ \\
\hline $\max \left\{\sigma_{P_{u}}\right\}$ & $16.3 \%$ & $5.7 \%$ & $1.8 \%$ \\
\hline
\end{tabular}

all time slots, users scheduled at the same time are allocated transmit powers which differ from less than $10 \%$. For $40 \%$ of them (those users located near the cell border, which are allocated more transmit power), this difference may even be less than $1 \%$. For users located around the AP, which possess the highest channel gains and are then allocated a weak power, the difference in transmit power may amount to $65 \%$. So, in the case where cells

Table 3 Proportion of scheduled users for which $\Delta<10 \%$ and $\Delta<1 \%$

\begin{tabular}{lcc}
\hline$\Delta$ & $<10 \%$ & $<1 \%$ \\
proportion of & $93 \%$ & $42 \%$ \\
scheduled users & $93 \%$
\end{tabular}


contain a different number of users, we may also assume that users scheduled at the same time are allocated the same power.

\section{Appendix 2}

A lower bound for the ergodic capacity $C_{\mathrm{erg}}^{\mathrm{WF}}\left(H_{\mathrm{pl}, 0}\right)$

In this Appendix, we establish a lower bound for the ergodic capacity $C_{\mathrm{erg}}^{\mathrm{WF}}\left(H_{\mathrm{pl}, 0}\right)$ given by formula (16):

$C_{\mathrm{erg}}^{\mathrm{WF}}\left(H_{\mathrm{pl}, 0}\right)=\underset{\substack{H_{\mathrm{fs}, 0}, H_{1}, H_{2}, \ldots, H_{N} \\ P_{1}, P_{2}, \ldots, P_{N}}}{\mathbb{E}}\left\{\log _{2}\left(1+\frac{P_{0}\left(H_{\mathrm{fs}, 0}\right) H_{\mathrm{pl}, 0} H_{\mathrm{fs}, 0}}{W+\sum_{n=1}^{N} P_{n} H_{n}}\right)\right\}$.

Since all r.v's $H_{\mathrm{fs}, 0}, H_{1}, H_{2}, \ldots, H_{N}, P_{1}, P_{2}, \ldots, P_{N}$, are independent of each other, operations to compute the expectation may be performed in any order; so focusing on the sole power $P_{n}$, we may rewrite (29) as follows:

$$
\begin{aligned}
& C_{\mathrm{erg}}^{\mathrm{WF}}\left(H_{\mathrm{pl}, 0}\right) \\
& =\underset{\substack{H_{\mathrm{fs}, 0}, H_{1}, H_{2}, \ldots, H_{N} \\
P_{1}, \ldots, P_{n-1}, P_{n+1}, \ldots, P_{N}}}{\mathbb{E}}\left\{\underset{P_{n}}{\mathbb{E}}\left\{\log _{2}\left(1+\frac{P_{0}\left(H_{\mathrm{fs}, 0}\right) H_{\mathrm{pl}, 0} H_{\mathrm{fs}, 0}}{W+\sum_{\substack{m=1 \\
m \neq n}}^{N} P_{m} H_{m}+P_{n} H_{n}}\right)\right]\right\} .
\end{aligned}
$$

Let us now define the function of one variable $f(x)$ as:

$$
f(x)=\log _{2}\left(1+\frac{P_{0}\left(H_{\mathrm{fs}, 0}\right) H_{\mathrm{pl}, 0} H_{\mathrm{fs}, 0}}{W+\sum_{\substack{m=1 \\ m \neq n}}^{N} P_{m} H_{m}+H_{n} x}\right), \quad x>0 .
$$

It can be easily shown that $f(x)$ is a convex function of the variable $x$, i.e., $\mathrm{d}^{2} f(x) / \mathrm{d} x^{2}>0, \forall x$. Using (31), the ergodic capacity (30) can be written

$$
C_{\text {erg }}^{\mathrm{WF}}\left(H_{\mathrm{pl}, 0}\right)=\underset{\substack{H_{\mathrm{f}, 0}, H_{1}, H_{2}, \ldots, H_{N} \\ P_{1}, \ldots, P_{n-1}, P_{n+1}, \ldots, P_{N}}}{\mathbb{E}}\left\{\underset{P_{n}}{\mathbb{E}}\left\{f\left(P_{n}\right)\right\}\right\} .
$$

Since computing (32) is too complex, we derive a lower bound for the ergodic capacity. To this end, we propose to approximate the r.v. $P_{n}$ by its mean value $\mathbb{E}\left\{P_{n}\right\}=$ $\mathbb{E}\left\{P_{0}\left(H_{\mathrm{fs}, 0}\right)\right\}$ (see Section 3.3). Since the function $f(x)$ is convex, we have, by Jensen's inequality [26], $\mathbb{E}\{f(x)\} \geq$ $f(\mathbb{E}\{x\})$, and the following holds:

$$
C_{\mathrm{erg}}^{\mathrm{WF}}\left(H_{\mathrm{pl}, 0}\right) \geq \underset{\substack{H_{\mathrm{f}, 0}, H_{1}, H_{2}, \ldots, H_{N} \\ P_{1}, \ldots, P_{n-1}, P_{n+1}, \ldots, P_{N}}}{\mathbb{E}}\left\{f\left(\underset{P_{n}}{\mathbb{E}}\left\{P_{n}\right\}\right)\right\}
$$

$$
=\underset{\substack{H_{\mathrm{fs}, 0}, H_{1}, H_{2}, \ldots, H_{N} \\ P_{1}, \ldots, P_{n-1}, P_{n+1}, \ldots, P_{N}}}{\mathbb{E}}\left\{\log _{2}\left(1+\frac{P_{0}\left(H_{\mathrm{fs}, 0}\right) H_{\mathrm{pl}, 0} H_{\mathrm{fs}, 0}}{W+\sum_{\substack{m=1 \\ m \neq n}}^{N} P_{m} H_{m}+\mathbb{E}\left\{P_{0}\left(H_{\mathrm{fs}, 0}\right)\right\} H_{n}}\right)\right\} .
$$

Applying the above procedure iteratively on $P_{m}, m=$ $1, \ldots, N, m \neq n$, and using (9) in association with (12)(14), yields:

$$
\begin{aligned}
& C_{\mathrm{erg}}^{\mathrm{WF}}\left(H_{\mathrm{pl}, 0}\right) \\
& \geq \underset{H_{\mathrm{fs}, 0}, H_{1}, \ldots, H_{N}}{\mathbb{E}}\left\{\log _{2}\left(1+\frac{P_{0}\left(H_{\mathrm{fs}, 0}\right) H_{\mathrm{pl}, 0} H_{\mathrm{fs}, 0}}{W+\mathbb{E}\left\{P_{0}\left(H_{\mathrm{fs}, 0}\right)\right\} \sum_{n=1}^{N} H_{n}}\right)\right\} \\
& =\underset{H_{\mathrm{fs}, 0}, G}{\mathbb{E}}\left\{\log _{2}\left(1+\frac{\left[L H_{\mathrm{fs}, 0}-J\right]^{+} H_{\mathrm{pl}, 0}}{W+\min \left(P_{\max }, \frac{P^{*}}{H_{\mathrm{pl}, 0}}\right) G}\right)\right\} \\
& \triangleq C_{\mathrm{erg}}^{\mathrm{wf}}\left(H_{\mathrm{pl}, 0}\right) .
\end{aligned}
$$

\section{Endnotes}

${ }^{a}$ Taking sectorization into consideration would indeed lead to a totally different interference model (see e.g., [24]). ${ }^{\mathrm{b}}$ Since we focus on power gains only, the term power will then be omitted in subsequent paragraphs.

${ }^{\mathrm{c}}$ Note that considering a full-CSI scheme at the network level would not be a realistic scenario because of the enormous amount of information that a central unit would have to manage.

${ }^{\mathrm{d}}$ The notation $[x]^{+}$means $\max (0, x)$.

e In what follows, we write $P_{0}\left(H_{\mathrm{fs}, 0}\right)$ instead of $P_{0}$ to emphasize the fact that, for a fixed value of $H_{\mathrm{pl}, 0}, P_{0}$ depends on the instantaneous channel state $H_{\mathrm{fs}, 0}$.

\section{Competing interests}

The authors declare that they have no competing interests.

\section{Author details}

${ }^{1}$ Université Lille Nord de France, F-59000 Lille, France. ${ }^{2}$ UVHC, IEMN/DOAE, F-59313 Valenciennes, France. ${ }^{3}$ CNRS, UMR 8520, F-59650 Villeneuve d'Ascq, France. 
Received: 22 July 2011 Accepted: 15 January 2013

Published: 22 February 2013

\section{References}

1. TS 300 evolved universal terrestrial radio access (E-UTRA) and evolved universal terrestrial radio access networks (E-UTRAN); overall description; stage 2, 3GPP Std (2010)

2. IEEE $802.16 \mathrm{~m}$ system description documents (SDD) 0034r3, IEEE Std. Rev. r3 (June 2010). [Online]. Available: [http://www.ieee802.org/16/tgm/ docs/80216m-09_0034r3.zip]

3. V Corvino, D Gesbert, R Verdone, in Proc. Wireless Communications and Networking Conference. A novel distributed interference mitigation technique using power planning, (Budapest, 2009), pp. 1-6

4. R Schoenen, F Qin, in Proc. 5th Int. Conf. on Wireless Communications, Networking and Mobile Computing. Adaptive power control for $4 \mathrm{G}$ OFDMA systems on frequency selective fading channels, (Beijing, 2009), pp. 1-6

5. G Boudreau, J Panicker, N Guo, R Chang, N Wang, S Vrzic, Interference coordination and cancellation for $4 \mathrm{G}$ networks. IEEE Commun. Mag. 47(4), 74-81 (2009)

6. N Himayat, STalwar, A Rao, R Soni, Interference management for $4 \mathrm{G}$ cellular standards [WiMAX/LTE UPDATE]. IEEE Commun. Mag. 48(8), 86-92 (2010)

7. TAli-Yahiya, $\mathrm{H}$ Chaouchi, Fractional frequency reuse for hierarchical resource allocation in mobile WiMAX networks. EURASIP J. Wirel. Commun. Network. 2010, 1-7 (2010)

8. W Fu, Z Tao, J Zhang, DP Agrawal, in Proc. IEEE Int. Conf. on Wireless Communications, Networking and Mobile Computing. Clustering based fractional frequency reuse and fair resource allocation in multi-cell networks, (Cape Town, 2010), pp. 1-5

9. K Kim, Interference mitigation in wireless communications. Ph.D. dissertation, School of Electrical and Computer Engineering, Georgia Institute of Technology (Atlanta, 2005)

10. F Wang, A Ghosh, C Sankaran, PJ Fleming, F Hsieh, SJ Benes, Mobile WiMAX systems: performance and evolution. IEEE Commun. Mag. 46(10), 41-49 (2008)

11. C Sankaran, F Wang, G Amitava, in Proc. IEEE 69th Vehicular Technology Conference, VTC Spring. Performance of frequency selective scheduling and fractional frequency reuse schemes for WiMAX, (Barcelona, 2009), pp. 1-5

12. ZXie, B Walke, in Proc. 3rd International Conference on New Technologies, Mobility and Security. Enhanced fractional frequency reuse to increase capacity of OFDMA systems, (Cairo, 2009), pp. 234-238

13. Y Zhou, N Zein, in Proc. IEEE 67th Vehicular Technology Conference, VTC Spring. Simulation study of fractional frequency reuse for mobile WiMAX, (Marina Bay, 2008), pp. 2592-2595

14. TD Novlan, RK Ganti, A Ghosh, JG Andrews, Analytical evaluation of Fractional Frequency Reuse for OFDMA cellular networks. IEEE Trans. Wirel. Commun. 12, 4294-4305 (2011)

15. N Markovitch, Nonparametric Analysis of Univariate Heavy-Tailed Data: Research and Practice, Wiley Series in Probability and Statistics, Wiley-Interscience, 1 edition. (John Wiley \& Sons Ltd, The Atrium, Southern Gate, Chichester, West Sussex PO19 8SQ, England, 2007)

16. B Pijcke, M Zwingelstein-Colin, M Gazalet, M Gharbi, P Corlay, An analytical model for the intercell interference power in the downlink of wireless cellular networks. EURASIP J. Wirel. Commun. Netw. 2011, 1-20 (2011)

17. SS Szyszkowicz, Interference in cellular networks: Sum of lognormals modeling (2007). Ph.D. dissertation, Carleton University, Ottawa, Ontario, Canada

18. A Goldsmith, Wireless Communications. (Cambridge University Press, 40 West 20th street, New-York, NY 10011-4211, USA, 2005)

19. SG Kiani, D Gesbert, Optimal and distributed scheduling for multicell capacity maximization. IEEE Trans. Wirel. Commun. 7, 288-297 (2008)

20. B Pijcke, M Zwingelstein-Colin, M Gazalet, M Gharbi, P Corlay, F-X Coudoux, in Proc. European Wireless Technology Conference. Contribution to the interference power modeling in cognitive radio networks, (Paris, 2010), pp. 137-140
21. A Goldsmith, P Varaiya, Capacity of fading channels with channel side information. IEEE Trans. Inf. Theory. 43(6), 1986-1992 (1997)

22. European Cooperative in the Field of Science and Technical Research EURO-COST 231, Urban transmission loss models for mobile radio in the 900 and $1800 \mathrm{MHz}$ bands. The Hague, Tech. Rep., September (1991) rev. 2

23. T Novlan, J Andrews, I Sohn, R Ganti, A Ghosh, in Proc. IEEE Global Telecommunications Conference. Comparison of fractional frequency reuse approaches in the OFDMA cellular downlink, (Miami, 2010), pp. 1-5

24. D Tse, P Viswanath, Fundamentals of Wireless Communication. (Cambridge University Press, The Edinburg Building, Cambridge CB2 8RU, UK, 2005)

25. N Balakrishnan, CR Rao, Handbook of Statistics 16 -Order Statistics: Theory \& Methods, Elsevier Science, 1 edition. (ELSEVIER SCIENCE B.V, Sarah Burgerhartstraat 25, P.O . Box 211, 1000 AE Amsterdam, The Netherlands, 1998)

26. TM Cover, JA Thomas, Elements of Information Theory 2nd Edition (Wiley Series in Telecommunications and Signal Processing), Wiley-Interscience; 2 edition. (John Wiley \& Sons, Inc., Hoboken, New Jersey, 2006)

doi:10.1186/1687-1499-2013-48

Cite this article as: Pijcke et al:: An accurate performance analysis of an FFR scheme in the downlink of cellular systems under large-shadowing effect. EURASIP Journal on Wireless Communications and Networking 2013 2013:48.

\section{Submit your manuscript to a SpringerOpen ${ }^{\mathcal{O}}$ journal and benefit from:}

- Convenient online submission

- Rigorous peer review

- Immediate publication on acceptance

- Open access: articles freely available online

- High visibility within the field

- Retaining the copyright to your article

Submit your next manuscript at $\gg$ springeropen.com 